\title{
Facile in situ Lithiation and Sodiation Observation in TEM Employing MF (M=Li,
} Na)

Jae Yeol Park ${ }^{1}$, Joon Ha Chang ${ }^{1}$, Sung Joo Kim ${ }^{1}$, Hyeon Kook Seo ${ }^{1}$ and Jong Min Yuk ${ }^{1}$

1. Department of Materials Science \& Engineering, Korea Advanced Institute of Science and Technology (KAIST), Daejeon and Republic of Korea.

Various techniques were suggested for real time observation of alkali ion ( $\mathrm{Li}$ and $\mathrm{Na}$ ) storage into active materials in transmission electron microscope (TEM). Huang et al. employed a biasing holder for potentiostatic alkali ion storage [1]. Yuk et al. introduced graphene liquid cell TEM (GLC-TEM) [2-6]. However, the electrochemical holder requires a substantial expense for the holder, and sample preparation is relative inconvenient. Although GLC-TEM technique provides reducing environment for alkali ion storage in liquid electrolyte, alkali element amount in GLC is insufficient for complete reaction [2-6].

Hence, here, we report facile in-situ technique for alkali ion storage employing $\mathrm{MF}(\mathrm{M}=\mathrm{Li}, \mathrm{Na})$. To prepare TEM samples, active materials and MF are dispersed in ethanol. They are dropped on graphenecoated $\mathrm{Au}$ grid. $\mathrm{MF}$ particles easily decomposes by electron beam irradiation generating the alkali metals [7]. The alkali metals directly react with active materials [8]. The alkali ion storage process into an active material is well descripted in Figure 1. MF provides sufficient alkali metal for complete reaction. As example cases, we present lithiation and sodiation of $\mathrm{CuS}$ and perform comparison study between them (Figure 2). CuS experiences displacement reaction in lithiation forming copper dendrite outside crystalline $\mathrm{Li}_{2} \mathrm{~S}$ matrix. On the other hand, it forms $\mathrm{Cu} / \mathrm{Na}_{2} \mathrm{~S}$ composite with uniformly distributed $\mathrm{Cu}$ in $\mathrm{Na}_{2} \mathrm{~S}$ matrix (Figure 2).

We believe this work provides invaluable insights for studying in-situ alkali ion storage mechanism into various active materials with the facile method [9].

\section{References:}

[1] JY Huang et al., Science 10 (2010), p. 1515.

[2] JM Yuk et al., ACS Nano 8 (2014), p. 7478.

[3] JH Chang et al., ACS Omega 2 (2017), p. 6329.

[4] JY Cheong et al., Nano Energy 25 (2016), p. 154.

[5] JY Cheong et al., Microscopy and Microanalysis 23 (2017), p. 1107.

[6] JH Chang et al., Journal of Visualized Experiments (2019), e58676.

[7] J Ghatak et al., Nanoscale 4 (2012), p.1754.

[8] JY Park et al., Nature Communications 9 (2018), p. 922.

[9] This work was supported by National Research Foundation of Korea (NRF) grant funded by the Korea government (MSIP; Ministry of Science, ICT \& Future Planning) (NRF-2018R1C1B6002624), which provided support for graphene growth and TEM sample preparation; NRF grant funded by Korea government (MSIP; Ministry of Science, ICT \& Future Planning) (NRF-2018M3A7B4065625), which provided support for electrode materials preparation; Nano·Material Technology Development Program through the National Research Foundation of Korea (NRF) funded by the Ministry of Science, ICT and Future Planning (2009-0082580), which provided support for TEM characterization; J.Y.P specially 
thanks NRF funded Korea government (MSIP; Ministry of Science, ICT \& Future Planning) for scholarship support through Global Ph.D. Fellowship Program (NRF-2018H1A2A1060105).

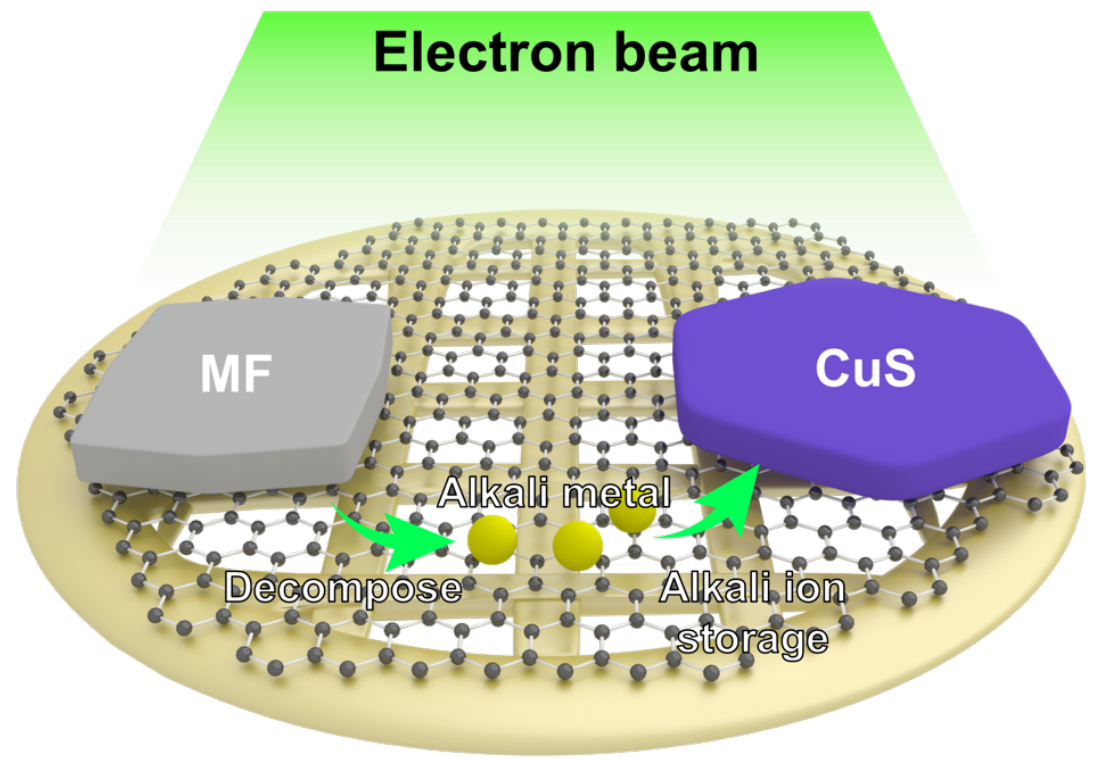

Figure 1. Schematic describing facile in-situ alkali ion storage method employing $\mathrm{MF}(\mathrm{M}=\mathrm{Li}, \mathrm{Na})$.

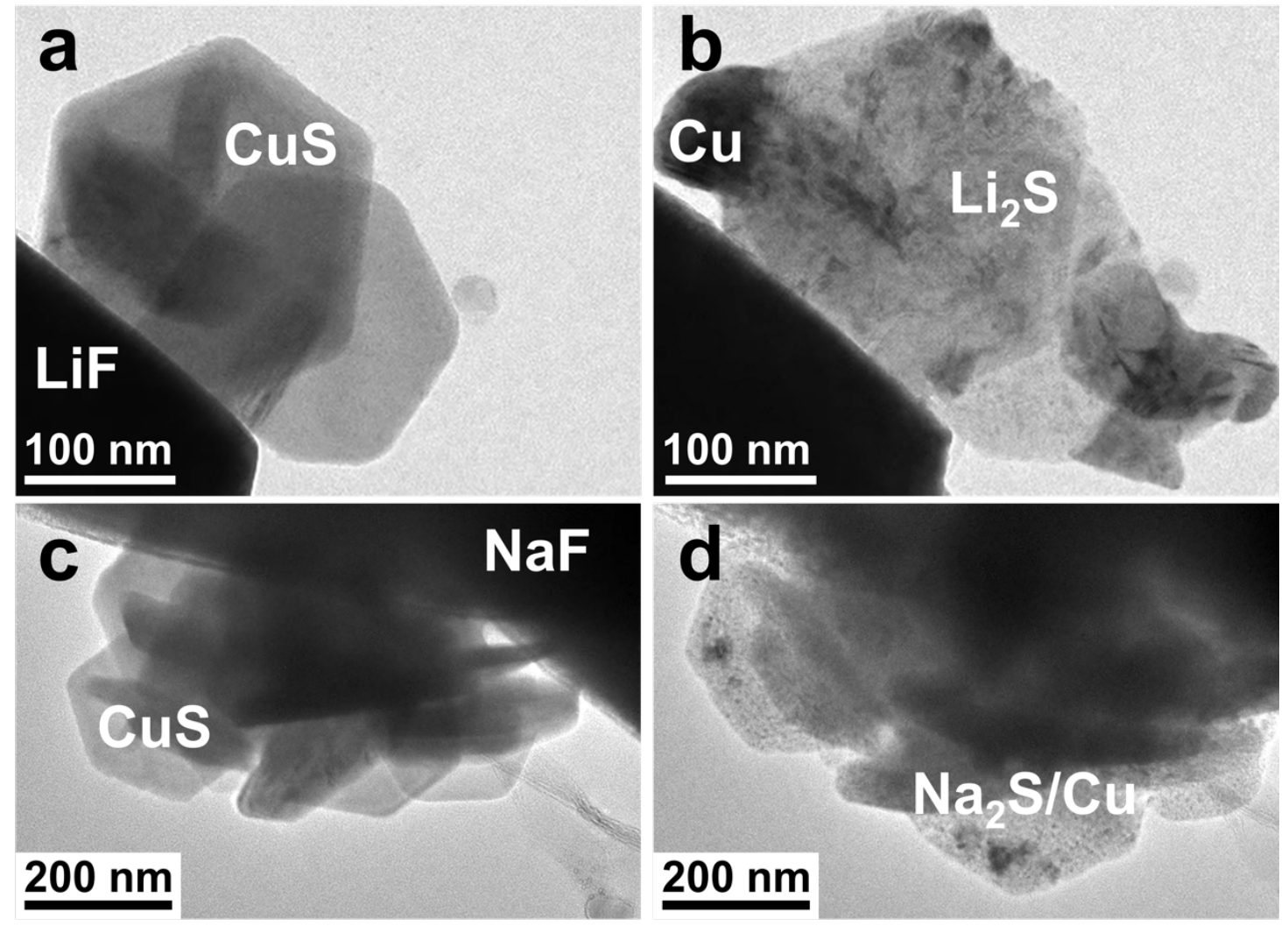

Figure 2. Example cases for lithium and sodium storages into CuS nanoplates. Low magnification TEM images of before (a) lithiation and (c) sodiation, and after (b) lithiation and (d) sodiation. 